\title{
Resultados do tratamento das cicatrizes queloideanas com cirurgia e imiquimode 5\% creme: um estudo prospectivo
}

Results of keloids treatment with surgery and iniquimod 5\% cream: a prospective study

Álisson YOSHIHARU

UMEMURA $^{1}$

Flávio Augusto Flório

Stillitano de Orgaes ${ }^{1}$

Newton José Borba

$\mathrm{CANICOBA}^{2}$

Patrícia Pinheiro Dorsa ${ }^{1}$

JuLiano GuARIZZO ${ }^{3}$

Hamilton Aleardo

GONELLA $^{4}$

Trabalho realizado no Conjunto Hospitalar de Sorocaba no Serviço de Cirurgia Plástica do Departamento de Cirurgia do Centro de Ciências Médicas e Biologicas da PUC - SP, Sorocaba, SP, Brasil.

Trabalho recebeu menção honrosa no Prêmio Victor Spina 2010.

Trabalho apresentado para ascensão à categoria de membro titular da

SBCP.

Artigo submetido pelo SGP (Sistema de Gestão de Publicações) da RBCP

Artigo recebido: 9/1/2011 Artigo aceito: 25/2/2011

\begin{abstract}
RESUMO
Introdução: O tratamento do queloide representa um desafio terapêutico, com resultados muitas vezes frustrantes. Terapias adjuvantes mais eficazes são necessárias para reduzir as altas taxas de recidivas. Essa pesquisa é baseada em uma modalidade conjunta com a aplicação de imiquimode $5 \%$ creme associada à cirurgia, tendo como objetivo a avaliação do tratamento e recidiva da cicatriz queloidiana. Método: Estudo prospectivo que avaliou 14 pacientes em um total de 25 queloides, com um seguimento mínimo de 6 meses. Foi realizada excisão intralesional e iniciada a aplicação do imiquimode $5 \%$ creme no dia da cirurgia e mantido por dois meses. A avaliação da cicatriz foi analisada por meio de uma Escala de Avaliação (paciente e um observador - regente de serviço de Cirurgia Plástica), sinais de recidiva e também do tamanho da cicatriz no pré e pós-operatório. Resultados: Após o acompanhamento de 6 meses, houve melhora da cicatriz por parte do paciente em $72 \% \mathrm{e}$ para o observador em $88 \%$, tendo uma taxa de recidiva de $8 \%$ e redução da área cicatricial $(\mathrm{p}=0,0053)$. Conclusão: $\mathrm{O}$ uso do Imiquimode $5 \%$ creme no pós-operatório da cirurgia de ressecção do queloide diminuiu significativamente sua recidiva na avaliação de 6 meses.
\end{abstract}

Descritores: Queloide. Recidiva. Aminoquinolinas.

\begin{abstract}
Introduction: The treatment of keloids represents a therapeutic challenge, with results often disappointing. More effective adjuvant therapies are needed to reduce high rates of relapse. This research is based on a combined modality with application of imiquimod $5 \%$ cream combined with surgery, having as objective the evaluation of treatment and recurrence of keloid scar. Methods: This prospective study evaluated 14 patients at a total of 25 keloids, with a minimum follow up of 6 months. Intralesional excision was performed and initiated the application of imiquimod 5\% cream on the day of surgery and continued for two months. The evaluation of the scar was analyzed by an Assessment Scale (patient and an observer ruler of service Plastic Surgery), signs of relapse and also the size of the scar in the pre-and postoperatively. Results: After 6 months of follow up, patients showed an improvement of $72 \%$ and $88 \%$ in the ruler's analysis, with a recurrence rate of $8 \%$ and reduction of scar area $(p=0.0053)$. Conclusion: The use of Imiquimode $5 \%$ cream in the post surgery for resection of the keloid recurrence decreased significantly in their evaluation of 6 months.
\end{abstract}

Keywords: Keloid. Recurrence. Aminoquinolinaes.

1. Cirurgião Plástico, Membro Especialista da Sociedade Brasileira de Cirurgia Plástica (SBCP), Serviço de Cirurgia Plástica Prof. Linneu Mattos Silveira PUC-Sorocaba, Sorocaba, SP, Brasil.

2. Cirurgião Plástico; Membro Titular da SBCP, Serviço de Cirurgia Plástica Prof. Linneu Mattos Silveira PUC-Sorocaba, Sorocaba, SP, Brasil.

3. Médico Estagiário do Serviço de Cirurgia Plástica Prof. Linneu Mattos Silveira PUC-Sorocaba, Sorocaba, SP, Brasil.

4. Cirurgião Plástico, Membro Titular da Sociedade Brasileira de Cirurgia Plástica, Chefe do Serviço de Cirurgia Plástica Prof. Linneu Mattos Silveira PUC-Sorocaba, Sorocaba, SP, Brasil. 


\section{INTRODUÇÃO}

As cicatrizes hipertróficas e queloides são desordens fibroproliferativas, decorrentes de uma cicatrização anormal por alteração na migração e na proliferação de células, inflamação, aumento na síntese de citocinas e proteínas da matriz extracelular e remodelamento da matriz sintetizada ${ }^{1}$. Os queloides diferem da cicatriz hipertrófica por apresentarem tecido cicatricial ultrapassando os limites das lesões que os originaram, sem tendência a regressão ou por continuarem a progredir após seis meses de evolução $0^{2,3}$.

Comumente encontradas na prática cirúrgica, os queloides têm incidência que varia de $5 \%$ a $16 \%$ nas populações de risco, incluindo negros, hispânicos e asiáticos ${ }^{4}$.

Muitos esquemas de tratamento têm sido utilizados, como o corticoide intralesional, compressão, laserterapia e criocirurgia, mas os resultados apresentados são inconsistentes ${ }^{5}$.

A excisão cirúrgica isolada pode apresentar taxas de recorrência entre $45 \%$ e $100 \%{ }^{4,6}$. Em combinação com a radioterapia, é considerado o tratamento mais eficaz disponível para os queloides graves, de acordo com as recomendações clínicas internacionais sobre o manejo da cicatriz ${ }^{7}$, porém van de Kar et al. ${ }^{5}$ sugerem que a excisão cirúrgica combinada com a radioterapia deveria ser reservada como último recurso no tratamento dos queloides resistentes à terapia.

Imiquimode creme a $5 \%$ é um modificador da resposta imune que junto à indução e ativação de células natural killers, macrófagos e células de Langerhans, induzem no local a síntese e a liberação de citocinas, incluindo interferon (IFN)-alfa, IFN-gama, fator de necrose tumoral-alfa, e interleucinas 1, 6, 8 e 12, quando aplicados topicamente.

Há uma inibição dose-dependente dos recursos humanos na produção de colágeno por fibroblastos IFN-alfa e IFNgama. O IFN-gama diminui tanto a produção de colágeno como glicosaminoglicano. Com base na capacidade de IFNalfa $2 b$ para reduzir a recidiva do queloide, o presente estudo tem o objetivo de avaliar o tratamento combinado de cirurgia e imiquimode $5 \%$ creme, bem como sua prevenção.

\section{MÉTODO}

Foram incluídos no estudo, 14 pacientes com 25 queloides que se apresentaram em um ambulatório de Serviço de Cirurgia Plástica, de acordo com os critérios: ferida que não respeitava os limites da cicatriz e não regridiu espontaneamente ou progrediu após 6 meses de evolução; pacientes livres de tratamento por 2 meses; presença de cicatriz por pelo menos 1 ano; cicatrizes que pudessem ser excisadas e suturadas primariamente; indivíduos vulneráveis, como crianças e adolescentes, acompanhados de seu representante legal (Tabela 1).

\begin{tabular}{l|c}
\hline \multicolumn{2}{c}{ Tabela 1 - Características dos pacientes e dos queloides. } \\
\hline \multicolumn{1}{c}{ Característica } & N \\
\hline Sexo & 10 \\
Masculino & 4 \\
Feminino & \\
\hline Etnia & 7 \\
Branco & 3 \\
Pardo & 4 \\
Negro & $11-57$ \\
\hline Idade (mínimo e máximo) & \\
\hline Localização do queloide & 6 \\
Lóbulo da orelha & 1 \\
Outras regiões da orelha & 4 \\
Abdome & 2 \\
Ombro & 3 \\
Tórax & 3 \\
Membros superiores & 6 \\
Outros & \\
\hline Fator desencadeante & 7 \\
Queimadura & 6 \\
Brinco / Piercing & 4 \\
Sequela de infecção & 3 \\
Pós-operatório & 2 \\
Trauma & 2 \\
Desconhecido & 1 \\
Vacina & 8 \\
\hline Tratamento prévio (número de queloides) & \\
\hline Queloides múltiplos no mesmo paciente & \\
(número de queloides) & \\
\hline & \\
\hline & \\
\hline & \\
\hline
\end{tabular}

Todos os pacientes assinaram termo de consentimento livre esclarecido. Foram excluídos do estudo os pacientes que não cumprissem com o protocolo de tratamento, quando a análise histológica revelasse um diagnóstico diferente de cicatriz queloideana, grávidas e em amamentação, portadores de doença cardíaca, transplantados, usuários de drogas imunossupressoras ou presença de infecção próxima à cicatriz ou com necessidade de mais de um procedimento cirúrgico para exérese total da cicatriz.

Todos os queloides foram excisados intralesionalmente, com anestesia local (Xylestesin 2\% com adrenalina 1:200.000; Cristália), controle da hemostasia com eletrocautério e fechamento primário com fio incolor inabsorvível no subdérmico, para reduzir a tensão, e sutura cutânea com fio preto inabsorvível (Ethilon nylon; Ethicon). Nos defeitos localizados na orelha, foi realizada somente sutura cutânea com Ethilon. A sutura cutânea foi removida após 14 dias.

O imiquimode $5 \%$ creme foi iniciado em todos os pacientes no dia da excisão cirúrgica e mantido por dois meses. O seguimento de seis meses foi realizado (Figuras 1 a 3), havendo avaliação subjetiva da cicatriz por meio de 


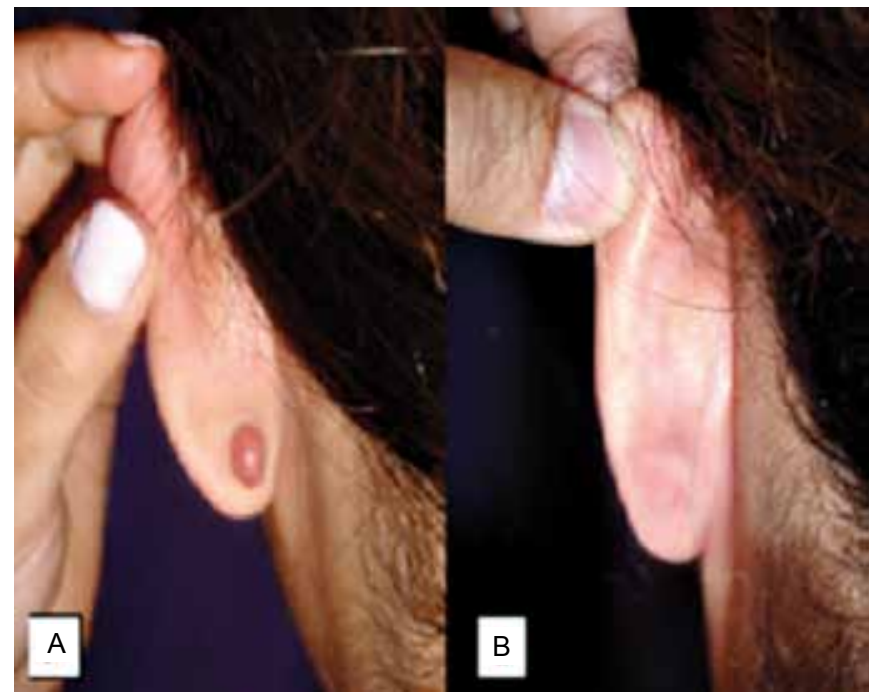

Figura 1-A: Paciente com queloide em lóbulo da orelha esquerda no pré-operatório. B: Após tratamento com exérese cirúrgica e Imiquimode 5\% creme, com pós-operatório de 24 semanas.

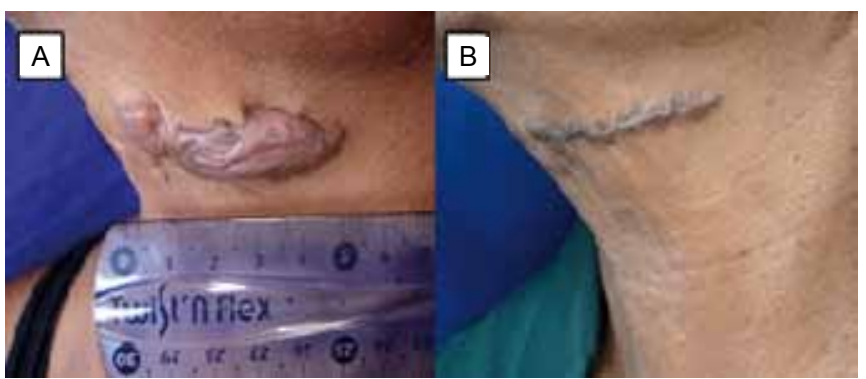

Figura 2-A: Paciente com queloide em região cervical no pré-operatório. B: Após tratamento com exérese cirúrgica e Imiquimode 5\% creme, com pós-operatório de 24 semanas.

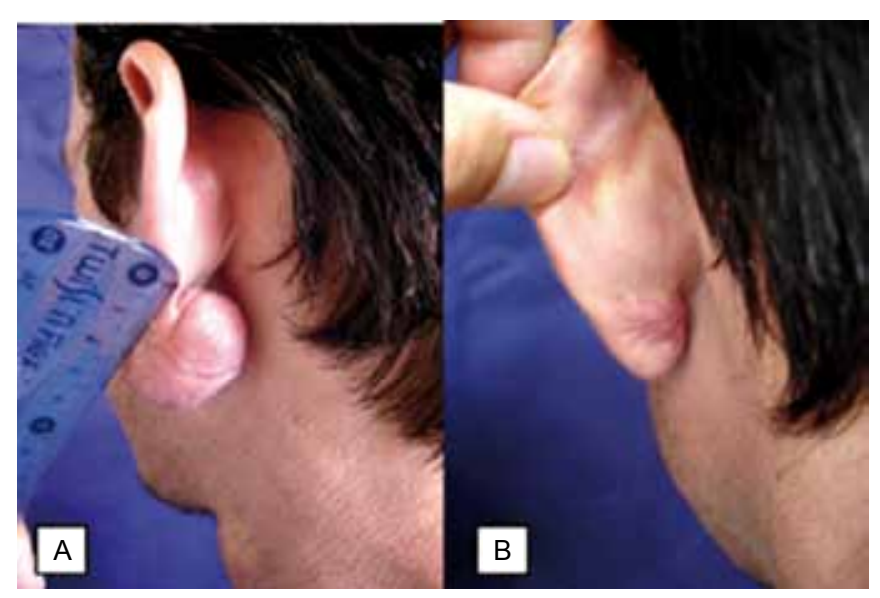

Figura 3 - A: Paciente com queloide em lóbulo da orelha esquerda no pré-operatório. B: Após tratamento com exérese cirúrgica e Imiquimode 5\% creme, com pós-operatório de 24 semanas. fotografias do pré e pós-operatórios por parte do paciente e um observador, que não participou de nenhuma cirurgia, sendo regente de um serviço de Cirurgia Plástica da SBCP, através de uma Escala de Avaliação. Tanto a escala do paciente como o do observador contém itens que são marcados numa escala de 1-10, onde o 1 indica "assemelhando-se à pele normal" e $10 \mathrm{a}$ "pior ferida possível". Para o observador, esses itens foram vascularização, pigmentação, superfície e impressão geral; para os pacientes, dor, prurido, cor, rigidez, espessura, irregularidade e impressão geral. Os pacientes responderam sobre a aparência da cicatriz como melhor, a mesma, ou pior, quer sejam ou não considerados o tratamento bem sucedido.

Foi feita uma análise descritiva para os dados dos pacientes, características do queloide, pós-operatório e avaliação da cicatriz na $24^{\mathrm{a}}$ semana. Foi aplicado um teste t-pareado para avaliar as áreas das cicatrizes do pré e pósoperatório, sendo fixado em $5 \%$ o índice de significância. Os sinais de recidiva foram avaliados pelo observador por meio de uma escala de cruzes (1-4), tendo como critério a elevação da cicatriz e sua extensão, além da margem cirúrgica original e ausência de recidiva (zero), considerada como cicatriz sem sinal de elevação ou extensão.

\section{RESULTADOS}

Foram avaliados 16 pacientes e 27 queloides; destes, em apenas 2 cicatrizes não ocorreu a confirmação histológica de cicatriz queloidiana, sendo excluídos do estudo. Quatorze pacientes com um total de 25 queloides foram avaliados para seguimento.

Em 100\% dos casos houve reações adversas nas duas primeiras semanas, variando de vermelhidão, ardência, dor, prurido, edema, descamação, diarreia, tontura até ulceração. Dos 25 queloides, foi avaliado o resultado pósoperatório pelo paciente e observador na $24^{\mathrm{a}}$ semana. Os resultados descritivos constam nas Tabelas 2 e 3 .

Para a cicatriz, a avaliação do paciente foi de melhora em $72 \%$ (18/25), igual à cicatriz prévia em $20 \%(5 / 25)$ e $8 \%(2 / 25)$ piora, sendo que do observador, houve melhora em 88\% (22/25) dos casos (Tabelas 4 e 5). Em relação aos sinais de recidiva, houve, por consideração do observador, ausência de recidiva em $16 \%$ (4/25) dos casos; $+/ 4+$ em $48 \%$; ++/4+ em $24 \%$; +++/4+ em $4 \%$ e $++++/ 4+$ em $8 \%$. $\mathrm{Na}$ avaliação das áreas das cicatrizes, nos dois momentos (pré-operatório e pós-operatório de 24 semanas), houve diminuição significativa com $\mathrm{p}=0,0053$ (Tabela 6). 


\begin{tabular}{l|c|c|c|c|c|c}
\hline \multicolumn{7}{c}{ Tabela 2 - Medidas descritivas para os itens de avaliação do paciente para 6 meses. } \\
\hline \multicolumn{1}{c|}{ Variável } & Média & Desvio padrão & N & Mínimo & Máximo & Mediana \\
\hline Dor & 1,92 & 1,38 & 25 & 1 & 7 & 1 \\
\hline Prurido & 2,96 & 2,41 & 25 & 1 & 10 & 3 \\
\hline Cor & 2,64 & 1,75 & 25 & 1 & 6 & 2 \\
\hline Rigidez & 3,48 & 2,04 & 25 & 1 & 7 & 3 \\
\hline Espessura & 4,56 & 3,22 & 25 & 1 & 10 & 3 \\
\hline Irregularidade & 3,40 & 2,25 & 25 & 1 & 9 & 4 \\
\hline Impressão geral & 3,76 & 2,55 & 25 & 1 & 9 & 3 \\
\hline
\end{tabular}

\begin{tabular}{l|c|c|c|c|c|c}
\hline \multicolumn{7}{c}{ Tabela 3 - Medidas descritivas para os itens de avaliação do observador para 6 meses. } \\
\hline \multicolumn{1}{c}{ Variável } & Média & Desvio padrão & N & Mínimo & Máximo & Mediana \\
\hline Vascularização & 1,08 & 0,40 & 25 & 1 & 3 & 1 \\
\hline Pigmentação & 1,72 & 1,06 & 25 & 1 & 5 & 1 \\
\hline Espessura & 2,04 & 1,17 & 25 & 1 & 5 & 2 \\
\hline Saliência & 1,92 & 1,04 & 25 & 1 & 4 & 2 \\
\hline Maleabilidade & 1,96 & 1,10 & 25 & 1 & 5 & 2 \\
\hline Superfície & 1,84 & 0,94 & 25 & 1 & 5 & 2 \\
\hline Impressão geral & 1,92 & 1,38 & 25 & 1 & 5 & 1 \\
\hline
\end{tabular}

\begin{tabular}{|c|c|c|c|}
\hline Item & Pontuacão & Frequência & Porcentagem \\
\hline \multirow[t]{6}{*}{ Dor } & 1 & 13 & 52 \\
\hline & 2 & 6 & 24 \\
\hline & 3 & 4 & 16 \\
\hline & 4 & 1 & 4 \\
\hline & 7 & 1 & 4 \\
\hline & Total & 25 & 100 \\
\hline \multirow[t]{8}{*}{ Prurido } & 1 & 11 & 44 \\
\hline & 2 & 1 & 4 \\
\hline & 3 & 6 & 24 \\
\hline & 4 & - & - \\
\hline & 5 & 5 & 20 \\
\hline & 8 & 1 & 4 \\
\hline & 10 & 1 & 4 \\
\hline & Total & 25 & 100 \\
\hline \multirow[t]{7}{*}{ Cor } & 1 & 11 & 44 \\
\hline & 2 & 2 & 8 \\
\hline & 3 & 4 & 16 \\
\hline & 4 & 2 & 8 \\
\hline & 5 & 5 & 20 \\
\hline & 6 & 1 & 4 \\
\hline & Total & 25 & 100 \\
\hline \multirow[t]{8}{*}{ Rigidez } & 1 & 3 & 12 \\
\hline & 2 & 8 & 32 \\
\hline & 3 & 3 & 12 \\
\hline & 4 & 6 & 24 \\
\hline & 5 & - & - \\
\hline & 6 & - & - \\
\hline & 7 & 5 & 20 \\
\hline & Total & 25 & 100 \\
\hline
\end{tabular}




\begin{tabular}{|c|c|c|c|}
\hline \multicolumn{4}{|c|}{ Continuação da Tabela 4} \\
\hline Espessura & 1 & 5 & 20 \\
\hline & 2 & 3 & 12 \\
\hline & 3 & 5 & 20 \\
\hline & 4 & 3 & 12 \\
\hline & 5 & - & - \\
\hline & 7 & 4 & 16 \\
\hline & 8 & - & - \\
\hline & 9 & 2 & 8 \\
\hline & 10 & 3 & 12 \\
\hline & Total & 25 & 100 \\
\hline \multirow[t]{8}{*}{ Irregularidade } & 1 & 7 & 28 \\
\hline & 2 & 3 & 12 \\
\hline & 3 & 2 & 8 \\
\hline & 4 & 7 & 28 \\
\hline & 5 & 4 & 16 \\
\hline & 8 & - & - \\
\hline & 9 & 2 & 8 \\
\hline & Total & 25 & 100 \\
\hline \multirow[t]{9}{*}{ Impressão geral } & 1 & 5 & 20 \\
\hline & 2 & 6 & 24 \\
\hline & 3 & 4 & 12 \\
\hline & 5 & 4 & 12 \\
\hline & 6 & 1 & 4 \\
\hline & 7 & 3 & 12 \\
\hline & 8 & - & - \\
\hline & 9 & 2 & 8 \\
\hline & Total & 25 & 100 \\
\hline \multirow[t]{4}{*}{ Cicatriz } & Igual & 5 & 20 \\
\hline & Melhor & 18 & 72 \\
\hline & Pior & 2 & 8 \\
\hline & Total & 25 & 100 \\
\hline
\end{tabular}

\begin{tabular}{c|c|c|c}
\hline \multicolumn{1}{c}{ Tabela 5 - Avaliação do observador para 6 meses. } \\
\hline Item & Pontuação & Frequência & Porcentagem \\
\hline Vascularização & 1 & 24 & 96 \\
\hline & 3 & 1 & $\mathbf{1 0 0}$ \\
\hline Pigmentação & Total & $\mathbf{2 5}$ & 60 \\
\hline & 1 & 15 & 16 \\
\hline Prurido & 2 & 4 & $\mathbf{2 0}$ \\
\hline & $\mathbf{3}$ & $\mathbf{5}$ & - \\
\hline & 5 & 1 & $\mathbf{1 0 0}$ \\
\hline & 6 & - & - \\
\hline & Total & $\mathbf{2 5}$ & 40 \\
\hline Espessura & 0 & - & 36 \\
\hline & 1 & 10 & 8 \\
\hline & 2 & 9 & $\mathbf{1 2}$ \\
\hline & 3 & 2 & 4 \\
\hline
\end{tabular}




\begin{tabular}{|c|c|c|c|}
\hline \multicolumn{4}{|c|}{ Continuação da Tabela 5} \\
\hline \multirow[t]{6}{*}{ Saliência } & 0 & - & - \\
\hline & 1 & 11 & 44 \\
\hline & 2 & 8 & 32 \\
\hline & 3 & 3 & 12 \\
\hline & 4 & 3 & 12 \\
\hline & Total & 25 & 100 \\
\hline \multirow[t]{8}{*}{ Maleabilidade } & 0 & - & - \\
\hline & 1 & 10 & 40 \\
\hline & 2 & 10 & 40 \\
\hline & 3 & 2 & 8 \\
\hline & 4 & 2 & 8 \\
\hline & 5 & 1 & 4 \\
\hline & 8 & - & - \\
\hline & Total & 25 & 100 \\
\hline \multirow[t]{8}{*}{ Superfície } & 1 & 10 & 40 \\
\hline & 2 & 11 & 44 \\
\hline & 3 & 3 & 12 \\
\hline & 4 & - & - \\
\hline & 5 & 1 & 4 \\
\hline & 7 & - & - \\
\hline & 10 & - & - \\
\hline & Total & 25 & 100 \\
\hline \multirow[t]{14}{*}{ Impressão geral } & 1 & 15 & 60 \\
\hline & 2 & 4 & 16 \\
\hline & 3 & 1 & 4 \\
\hline & 4 & 3 & 12 \\
\hline & 5 & 2 & 8 \\
\hline & 7 & - & - \\
\hline & 8 & - & - \\
\hline & 9 & - & - \\
\hline & 10 & - & - \\
\hline & Total & 25 & 100 \\
\hline & Igual & 1 & 4 \\
\hline & Melhor & 22 & 88 \\
\hline & Pior & 2 & 8 \\
\hline & Total & 25 & 100 \\
\hline
\end{tabular}

Tabela 6 - Comparação entre as áreas no pré e pós-operatório de 6 meses.

\begin{tabular}{l}
\hline Média \\
\hline Desvio padrão \\
\hline Observações \\
\hline p-valor \\
\hline
\end{tabular}

\begin{tabular}{l|c|}
\multicolumn{3}{|c}{ Tabela 6 - Comparação entre as áreas no pré e pós-o } \\
\hline Avaliação pré-operatória \\
3,116 & 2,793397 \\
\hline $\mathbf{0 , 0 0 5 3}$ & \\
\hline
\end{tabular}

\begin{tabular}{|c}
\hline 1,7312 \\
\hline 2,226421 \\
\hline 25 \\
\hline
\end{tabular}

\section{DISCUSSÃO}

Os queloides foram excisados e tratados com imiquimode $5 \%$ creme, agindo sobre a cicatriz durante 6 a 8 horas no período noturno à cirurgia e, retirado com água na manhã seguinte, com posterior uso de pomada tópica (neomicina/ bacitracina) por 2 meses. Este estudo se baseia na capacidade do IFN-alfa2b para reduzir a recidiva do queloide, quando usado imediatamente após a excisão, o que foi demonstrado por Berman \& Flores ${ }^{8}$, em 1997. Kaufman \& Berman ${ }^{9}$ examinaram os efeitos de imiquimode $5 \%$ creme, não havendo recorrência nas 24 semanas, entre os 11 queloides dos 10 
pacientes que completaram o estudo, uma taxa inferior ao relatado na literatura ${ }^{4,6}$. Resultados semelhantes foram relatados por Arellano e outros no Congresso Mundial de Dermatologia (Paris, 2002) ${ }^{10}$. Em nosso estudo, consideramos apenas $8 \%$ de recidiva $(++++/ 4+)$, já que as outras avaliações poderão mudar com a evolução mais tardia para uma cicatriz hipertrófica ou mesmo alargada. Em nossa pesquisa, os queloides tratados estão em várias localizações em detrimento dos estudos supracitados que avaliaram somente uma localização ${ }^{9}$. Alguns autores afirmam não haver diferença do prognóstico baseado na localização ${ }^{11,12}$, mas outros relatam diferenças clínicas significantes ${ }^{13,14}$, como os queloides do tórax e ombros devido à grande tensão nestas áreas, o que foi comprovado neste trabalho com $100 \%$ das recidivas, localizado no tórax.

Os queloides incluídos no trabalho foram histologicamente confirmados após a excisão, fato este comentado por Cosman $^{13}$, que notou diferenças nas taxas de recidiva nas cicatrizes sem confirmação histológica, havendo possibilidade de inclusão de cicatrizes hipertróficas nos estudos sem confirmação.

Há significativa variação no período de seguimento entre os estudos de queloide, muitos recomendam um mínimo de 1 ano $^{11,13}$, outros postulam até 2 anos $^{12,15}$. O trabalho exposto apresenta resultados com 6 meses de seguimento, o que poderia explicar a porcentagem reduzida de recidiva. Muitos autores falharam em definir como os seus resultados tiveram sucesso ou recidiva ${ }^{4,9,12}$, este estudo, além de usar uma escala de avaliação do resultado pós-operatória do paciente e um observador, também avaliou os sinais de recidiva e cálculo da área cicatricial no pré e pós-operatório, com redução significativa.

\section{CONCLUSÃO}

Nas 24 semanas, a porcentagem de recidiva após excisão do queloide e tratamento adjuvante com imiquimode 5\% demonstra que a terapia é promissora, apesar de haver necessidade de tempo maior de seguimento.

\section{REFERÊNCIAS}

1. Alves JCRR, Silva Filho AF, Pereira NA. Cicatrização patológica e seu tratamento. In: Mélega JM, ed. Cirurgia plástica fundamentos e arte - princípios gerais. Rio de Janeiro:Medsi;2002. p.15-24.

2. Figueiredo JCA, Junior FCO, Zampar AG, Mélega JM. Quelóide: fatores de influência prognóstica. Rev Bras Cir Plást. 2008;23(4):274-80.

3. Ogawa R, Mitsuhashi K, Hyakusoku H, Miyashita T. Postoperative electron-beam irradiation therapy for keloids and hypertrophic scars: retrospective study of 147 cases followed for more than 18 months. Plast Reconstr Surg. 2003;111(2):547-53.

4. Berman B, Villa A. Imiquimod $5 \%$ cream for keloid management. Dermatol Surg. 2003;29(10):1050-1.

5. van de Kar AL, Kreulen M, van Zuijlen PP, Oldenburger F. The results of surgical excision and adjuvant irradiation for therapy-resistant keloids: a prospective clinical outcome study. Plast Reconstr Surg. 2007;119(7):2248-54.

6. Rosen DJ, Patel MK, Freeman K, Weiss PR. A primary protocol for the management of ear keloids: results of excision combined with intraoperative and potoperative steroid injections. Plast Reconstr Surg. 2007;120(5):1395-400.

7. Mustoe TA, Cooter RD, Gold MH, Hobbs FD, Ramelet AA, Shakespeare PG, et al; International Advisory Panel on Scar Management. International clinical recommendations on scar management. Plast Reconstr Surg. 2002;110(2):560-71.

8. Berman B, Flores F. Recurrence rates of excised keloids treated with postoperative triamcinolone acetonide injections or interferon alpha- $2 \mathrm{~b}$ injections. J Am Acad Dermatol. 1997;37(5 Pt 1):755-7.

9. Kaufman J, Berman B. Topical application of imiquimod $5 \%$ cream to excision sites is safe and effective in reducing keloid recurrences. J Am Acad Dermatol. 2002;47:S209-11.

10. Clark J. Imiquimod vs. interferon: cream matches injection in terms of eliminating keloid recurrence while showing no adverse events. Dermatol Times. 2002:15-6.

11. Ollstein RN, Siegel HW, Gillooley JF, Barsa JM. Treatment of keloids by combined surgical excision and immediate postoperative X-ray therapy. Ann Plast Surg. 1981;7(4):281-5.

12. Kovalic JJ, Peres CA. Radiation therapy following keloidectomy: a 20year experience. Int J Radiat Oncol Biol Phys. 1989;17(1):77-80.

13. Cosman B. The surgical treatment of keloids. Plast Reconstr Surg. 1961;27:335.

14. EnhamreA, Hammar H. Treatment of keloids with excision and postoperative X-ray irradiation. Dermatologica. 1983;167(2):90-3.

15. Norris JE. Superficial X-ray therapy in keloid management: a retrospective study of 24 cases and literature review. Plast Reconstr Surg. 1995;95(6):1051-5.
Correspondência para:
Álisson Yoshiharu Umemura

Rua Benedito Ferreira Telles, 341, apto 82, bloco B - Jd. Simus - Sorocaba, SP, Brasil - CEP: 18055-270

E-mail: alissonumemura@ig.com.br 